Broman-Fulks, J. J., Berman, M. E., Martin, H. M., Marsic, A., Harris, J. A. (2009). The phenomenon of declining anxiety sensitivity scores: A controlled investigation. Depression and Anxiety, 26(1): E1-E9. Published by Wiley (ISSN: 1520-6394). DOI: 10.1002/da.20436 The definitive version is available at www3.interscience.wiley.com

\title{
Phenomenon of Declining Anxiety Sensitivity Scores: A Controlled Investigation
}

\author{
Joshua J. Broman-Fulks, Ph.D., Mitchell E. Berman, Ph.D., H. Marty Martin, B.A.,
} Angelica Marsic, B.A., and Jessica A. Harris

\begin{abstract}
Background: Repeated administration of anxiety sensitivity measures can often produce declining scores, even in ostensible control groups, which is a significant concern for researchers. The reasons for these changes are as yet unknown, but could be because of regression to the mean in samples selected on extreme scores, exposure to general information about anxiety contained in psychiatric interviews, or mere exposure to anxiety sensitivity information. Methods: This study sought to experimentally evaluate these potential explanations using a comprehensive measure of anxiety sensitivity and its subcomponents, a non-anxiety sensitivity measure (self-esteem), and participants representing the full spectrum of anxiety sensitivity. Results: Results indicated significant decreases in anxiety sensitivity scores (but not self-esteem scores) that could not be accounted for by regression to the mean or exposure to information about anxiety in general. Conclusions: Several potential explanations for these findings are reviewed and implications for research study designs are discussed.
\end{abstract}




\section{INTRODUCTION}

Anxiety sensitivity is the persistent fear of anxietyrelated symptoms arising from beliefs that such

symptoms are associated with negative physical, social, or psychological consequences.[1] Anxiety sensitive individuals have a tendency to catastrophize relatively benign anxiety-related physiological sensations,[1] such as believing that heart palpitations are a sign of an imminent heart attack. Over the past 20 years, considerable research has accumulated demonstrating that anxiety sensitivity plays an important role in the development and maintenance of various forms of psychopathology, particularly anxiety and panic [e.g.,].[2,3]

Anxiety sensitivity was initially conceptualized as a dispositional construct, denoting a consistent tendency to respond fearfully when anxiety-related symptoms are present.[1] Early research into the psychometric properties of the Anxiety Sensitivity Index [ASI],[4] which has been the predominant measure of anxiety sensitivity, indicated that scores on the measure tend to predict later scores, with a test-retest correlation of 0.71 across a 3-year interval.[2] Subsequent studies have shown test-retest correlations of similar magnitude in both adult and adolescent samples assessed over varying intervals, ranging from 2 weeks to 4 years [e.g.,.].[5,6] Although high test-retest correlations provide some support for the "stability" of scores on the measure, and given sufficient temporal distance between testing administrations can be interpreted to mean that a construct is dispositional in nature, test-retest correlations provide, in part, an estimate of the degree to which the relative position of scores stays the same across time. Recall that adding or subtracting a constant from every score on the second administration of a measure will not affect the magnitude of the correlation coefficient. Hence, it is possible to have significant mean differences in scores across administrations, whereas at the same time having high test-retest correlations. Recently, anxiety sensitivity researchers have begun to note exactly such a phenomenon.

In an attempt to examine the stability of anxiety sensitivity scores,[5] administered the ASI to a large sample of adolescents yearly across a 4-year period. Although results indicated significant ASI test-retest correlations across the 4 years, overall mean ASI scores declined at each assessment. Cluster analyses were 
conducted to examine the patterns of ASI scores over time, and more than $80 \%$ of participants were classified as having "stable low" or "stable high" anxiety sensitivity. However, further examination of the "stable low" and "stable high" groups revealed decreases in ASI scores of greater than 0.5 and $1.0 \mathrm{SD}$ for the two groups, respectively, over the 4-year period. Thus, despite the high test-retest reliability of anxiety sensitivity scores, participants were reporting significantly less fear of anxiety symptoms at each assessment session. Several recent empirical studies of panic prevention or treatment programs have also noted significant reductions in anxiety sensitivity scores among individuals assigned to ostensible control conditions. In one study,[7] attempted to evaluate the efficacy of a panic prevention program in individuals who had been identified as at risk for panic disorder (i.e., experienced at least one panic attack in the past year and had ASI scores 415). Participants were randomly assigned to either 6 months of cognitive-behavioral treatment, which included psychoeducation and interoceptive exposure, or to a wait-list control condition. All participants were administered a diagnostic assessment before beginning their respective treatment protocols. Results indicated that there were no ASI differences between the treatment and control groups, and both groups showed about a 1 SD decline in ASI scores at posttesting. The authors attributed ASI score reductions in the wait-list control condition to non-specific factors, such as reassurance, support, or the expectation of receiving treatment. However, because participants were pre-selected based on extreme scores, regression to the mean could not be ruled out as a plausible alternative explanation.

Schmidt et al. [2007][8] noted similar issues in a recent attempt to evaluate a longitudinal prevention program for individuals at risk for anxiety and other axis I disorders. Participants with high ASI scores (41.5 SD above the mean) who did not meet current criteria for an axis I disorder (as assessed by a structured diagnostic interview) were randomized to either Anxiety Sensitivity Amelioration Training (ASAT) or control. Participants in the ASAT group watched a video presentation that provided psychoeducation regarding anxiety, stress, and anxiety sensitivity as well as behavioral exercises designed to correct maladaptive interoceptive conditioning. The control condition received general health and nutrition information via a computer presentation of comparable length to the ASAT presentation. Although results 
indicated that the ASAT group reported greater decreases in ASI scores immediately after the presentations, the control condition also reported declines in anxiety sensitivity (approximately $0.5 \mathrm{SD}$ ), and the group differences were not maintained at 1 and 2-year follow-ups.

On the basis of the observation that structured diagnostic interviews are frequently administered to experimental and control participants in anxiety sensitivity intervention studies,[9] suggested that reductions in anxiety sensitivity may occur in response to the diagnostic interviews. Specifically, anxiety disorder interviews may provide indirect normalizing information regarding common anxiety-related physical symptoms via the assessment of panic attack criteria, which may, in turn, lead individuals with high anxiety sensitivity to reevaluate their catastrophic beliefs about the consequences of such symptoms. To test this hypothesis,[9] used a non-experimental design to compare two separate "cohorts" of high anxiety sensitivity individuals (ASI scores 423) with respect to changes in anxiety sensitivity scores over a 2-week period. One cohort received a diagnostic interview (Structured Clinical Interview for DSM-IV-SCID-IV) as a function of participation, whereas the second one did not. Results indicated that the cohort exposed to a structured interview reported a significant decline in anxiety sensitivity scores at a 2-week follow-up assessment, but the cohort that did not receive a structured interview exhibited no change in anxiety sensitivity scores. A significant limitation of this study, however, was that the research design involved ex post facto analyses of two cohort samples that were derived from separate studies with seemingly different initial aims (one cohort was derived from a treatment outcome study) and somewhat different inclusion criteria directly relevant to anxiety sensitivity. Both cohorts were also pre-selected based on elevated anxiety sensitivity scores, which provide a limited picture of anxiety sensitivity fluctuations and the ability to isolate potential regression to the mean effects. Moreover, the psychiatric interview included exposure to both anxiety-related and non-anxiety-related symptoms. Thus, it is not clear whether exposure to anxiety information per se was responsible for the decline in scores observed. Finally, because data from the cohorts were presumably collected at different time points, one cannot rule out the possibility that the changes in anxiety sensitivity observed in the first cohort, or lack of changes in the second, are not better 
accounted for by exposure to extraneous variables associated with their different histories.

The phenomenon of declining anxiety sensitivity scores raises important questions concerning the conceptualization and stability of anxiety sensitivity, as well as the potential clinical utility of administering diagnostic interviews to patients. This study is, to the best of our knowledge, the first to experimentally examine the effects of repeated instrument administration of anxiety sensitivity measures on anxiety sensitivity scores. In contrast to earlier studies, participants in this study were not pre-selected based on extreme anxiety sensitivity scores. Also, to build on previous research, an expanded measure of anxiety sensitivity that also allowed for an assessment of changes in the subcomponents of anxiety sensitivity was used and compared to a non-anxiety sensitivity measure. An analysis of subcomponent changes may aid in discriminating the specific aspects of anxiety sensitivity that are affected by repeated administrations of anxiety sensitivity measures. Also, recall that researchers have speculated that exposure to general information about anxiety in the context of a structured diagnostic interview may be responsible for declines in anxiety sensitivity scores. To examine this possibility, the effects of administering an anxiety-related structured interview were compared with administration of a nonanxietyrelated structured interview and no interview.

In addition, we compared the effects of providing general information about anxiety indirectly via interview to direct provision of information about anxietyrelated symptoms via interview plus education. Finally, in contrast to previous studies,[7,9] we also assessed anxiety sensitivity immediately after the administration of the respective manipulations, and not just at a 2week follow-up, to establish a closer temporal link between the variables of interest and changes in anxiety sensitivity scores. Following the logic of,[9] exposure to a structured interview specific to anxiety disorders should produce greater declines in anxiety sensitivity scores compared to a non-anxiety-related interview and control condition. Furthermore, if the declines in anxiety sensitivity scores noted in previous reports are truly the result of participants receiving indirect normalizing information from anxiety-related diagnostic interviews, the direct provision of anxiety-related information in combination with an anxiety disorders diagnostic interview should provide even greater reductions in anxiety sensitivity scores. 


\section{METHODS}

\section{PARTICIPANTS}

The participants were 190 undergraduate students, ages 18 through 35 years (M519.36, SD52.02) who received course credit for their participation. The majority of participants were women (61.6\%) and Caucasian (92\%). Of the 190 participants who began the study, 20 failed to return to the laboratory to complete follow-up measures. Thus, the analyses that follow are based on the 170 completers, unless otherwise stated. The consent process and protocol were reviewed by the Institutional Review Board for the Protection of Human Subjects at the Appalachian State University.

\section{INSTRUMENTS}

Anxiety Sensitivity Index-Revised. The ASI-Revised[10] is a self-report questionnaire that assesses fear of anxiety and anxietyrelated sensations. The revised version of the ASI consists of 36 items (as opposed to 16 on the original ASI) that are rated on a 5-point Likert scale, ranging from "Very Little" to "Very Much." The ASI-R was designed to assess the major subcomponents of anxiety sensitivity in greater depth. The ASI-R has been shown to assess a higher-order general anxiety sensitivity factor, and four lower-order factors, which were labeled: (1) fear of respiratory symptoms, (2) fear of publicly observable anxiety reactions, (3) fear of cardiovascular symptoms, and (4) fear of cognitive dyscontrol.[10] Construct validity for the ASI-R has been established based on significant correlations with the original version of the ASI [r50.94].[10] The ASI-R has demonstrated excellent internal consistency (a50.95), with all 36 items showing adequate item-total correlations [M50.58, range50.40-0.71].[11] The ASI-R was selected for use in this study based on its well-documented psychometric properties and its ability to comprehensively assess the full spectrum of the anxiety sensitivity construct.

Rosenberg Self-Esteem Scale. The Rosenberg Self-esteem Scale [RSES][12] is a 10-item, self-report scale, designed to measure global self-esteem. Anxiety sensitivity and self-esteem have been shown to be negatively correlated[13] and both phenomena are conceptualized as relatively stable dispositional characteristics. Thus, the RSES was included in this study in an attempt to rule out general test reactivity as an explanation for the decline in anxiety sensitivity scores. The RSES consists of 10 items that are rated on a 4-point Likert scale, ranging from "Strongly Agree" to "Strongly Disagree." The RSES has demonstrated good reliability and validity across a large number of different sample groups [e.g.,].[14] Specifically, Cronbach a' scores have ranged between 
0.77 and 0.88 , and test-retest correlations have ranged between 0.82 and 0.85 across 1 and 2-week intervals, respectively [for further details, see].[15,16]

\section{Structured Clinical Interview for DSM-IV Axis I \\ Disorders. The SCID-IV Axis I Disorders [SCID-I][17] is a} semi-structured interview designed to assist trained raters in making reliable axis I diagnoses. In this study, only the anxiety disorders (SCID-A) and psychotic disorders modules (SCID-P) were used. The anxiety disorders module assesses for the following anxiety disorders: panic disorder, agoraphobia, specific phobias, social phobia, posttraumatic stress disorder, obsessive-compulsive disorder, and generalized anxiety disorder. The SCID-A has demonstrated good reliability, with k's ranging between 0.57 (for obsessive-compulsive disorder) and 0.88 [for posttraumatic stress disorder; e.g.,],[18] and validity. The SCID-P assesses for schizophrenia, schizophreniform, schizoaffective disorder, delusional disorder, brief psychotic disorder, and psychotic disorder that are not otherwise specified. The reliability and validity of the SCID-P have also been well documented, with the SCID-P leading to accurate psychotic disorder diagnoses and $\mathrm{k}$ estimates as high as 0.94.[19]

\section{PROCEDURE}

Participants completed the informed consent process and were randomly assigned to one of four conditions: (1) SCID-IV Anxiety Disorders Module (SCID-A), (2) SCID-A plus educational information concerning common physiological responses to stress, (3) a nonanxietyrelated structured interview (SCID-Psychotic Disorders Module-SCID-P), or (4) no interview/no information. Regardless of group assignment, all participants completed the ASI-R and RSES at two time points: (1) before completing the tasks associated with the respective experimental conditions and (2) at a 2-week follow-up appointment. The order of completion for the questionnaires was counterbalanced across participants. All participants also completed the ASI-R one additional time immediately after the experimental manipulation (e.g., completing the interview). Individuals assigned to the no interview/no information control condition were asked to wait in an empty research room for approximately 5 min before completing the ASI-R the second time. The purpose of the 5-min wait period was to provide the control condition with a comparable time frame between baseline and post-ASI-R measurements to those groups that were administered the SCID-A and SCID-P. Furthermore, one of the reasons for selecting the SCID-P as the nonanxietyrelated interview was that it is comparable to the SCID-A in average administration time (approximately 4-7 min depending on participant responses). 
Participants assigned to one of the conditions receiving the SCIDA or SCID-P were administered their respective SCID modules by a research assistant who had received advanced training in the administration of the SCID. The training protocol for the interviewers included a review of SCID materials, SCID administration observations of an experienced clinician, and satisfactory practice administrations to volunteers before working with research participants. The research assistant was not aware of participant scores on the self-report measures before SCID administration. In addition to completing the interview, participants in the SCID-A plus information group were provided with details about the physiology of anxiety and panic reactions via an electronic voice recording. The information they received was based on a slightly modified version of the psychoeducation information contained in the Mastery of Your Anxiety and Panic treatment manual[20] directly explaining the fight-flight response, the typical psychophysiological symptoms of a fear/panic response, and reasons why it is beneficial and adaptive for the organism to respond to danger in this manner.

\section{RESULTS}

The study hypotheses were tested using separate group by assessment session mixed-model analyses of variance (ANOVAs) for each of the dependent measures. The repeated measures factor for the ASI-R analyses had three levels (baseline, post, and followup), whereas RSE measurements were only taken at baseline and follow-up.

\section{COMPLETERS VERSUS NON-COMPLETERS}

Participants who completed the entire protocol (i.e., had baseline, post, and follow-up measurements) were compared with those who dropped out of the study to determine whether dropouts differed from completers in any meaningful way. On average, participants who failed to complete the study were more likely to be male, t(188)5_2.62, P5.01 and had lower levels of baseline anxiety sensitivity (M524.75 compared to completers M535.08), t(188)5_2.26, P5.03. All dropouts occurred between post and follow-up measurements. Thus, group differences in ASI-R change from baseline to post were examined to determine whether non-completers were reporting significantly different changes in ASI-R scores. Results indicated that completers and non-completers did not significantly differ on ASI-R score change from baseline to 
post, t(188)5_1.18, P5.24. Thus, although noncompleters reported lower anxiety sensitivity scores at baseline, they were reporting comparable decreases in anxiety sensitivity from baseline to post as those individuals who completed the entire study.

\section{BASELINE ANALYSES}

One-way ANOVAs and w2 analyses indicated that the groups were comparable at baseline on all demographic variables (all P4.10; see Table 1). In addition, no significant differences were noted between the groups on baseline levels of anxiety sensitivity (ASI-R, $F(3,169) 51.60, P 4.10)$. Baseline ASI-R scores (M535.1, SD519.7) were comparable to those of non-selected samples in previous reports [e.g., M525.7, SD519.6],[11] and substantially lower than ASI-R scores in clinical samples [e.g., M555.6, SD530.0].[21]

TABLE 1. Relevant demographic characteristics by condition

\begin{tabular}{|c|c|c|c|c|c|c|c|c|}
\hline \multirow[b]{2}{*}{ Variable } & \multicolumn{2}{|c|}{ SCID-A $(n=47)$} & \multicolumn{2}{|c|}{ SCID-A+Information $(n=42)$} & \multicolumn{2}{|c|}{ SCID-P $(n=42)$} & \multicolumn{2}{|c|}{ No interview $(n=39)$} \\
\hline & $N$ & Mean $(S D)$ & $N$ & Mean $(S D)$ & $N$ & Mean $(S D)$ & $N$ & Mean $(S D)$ \\
\hline Age & & $19.34(1.56)$ & & $19.52(1.94)$ & & $19.31(2.68)$ & & $19.51(2.24)$ \\
\hline \multicolumn{9}{|l|}{ Gender } \\
\hline Men & 17 & & 18 & & 14 & & 11 & \\
\hline Women & 30 & & 24 & & 28 & & 28 & \\
\hline \multicolumn{9}{|l|}{ Race } \\
\hline Caucasian & 43 & & 38 & & 38 & & 36 & \\
\hline African-American & 1 & & 0 & & 1 & & 1 & \\
\hline Asian & 1 & & 1 & & 1 & & 0 & \\
\hline Hispanic & 1 & & 3 & & 1 & & 0 & \\
\hline Biracial & 0 & & 0 & & 0 & & 2 & \\
\hline Other/missing & 1 & & 0 & & 1 & & 0 & \\
\hline
\end{tabular}

SCID-A, Structured Clinical Interview for DSM-IV Diagnoses-Anxiety Disorders Module; SCID-A+Information, Structured Clinical Interview for DSM-IV Diagnoses_Anxiety Disorders Module plus education regarding physiological responses to anxiety; SCID-P, Structured Clinical Interview for DSM-IV Diagnoses-Psychotic Disorders Module.

\section{TEST-RETEST CORRELATIONS}

Baseline scores on the outcome measures were correlated with their corresponding follow-up scores (and also at post for the ASI-R). Results indicated moderate to high test-retest correlations over a 2-week period for both of the outcome measures. Specifically, the ASI-R demonstrated the highest test-retest correlations, 
with baseline to post (r50.89), baseline to follow-up (r50.83), and post to follow-up (r50.91). RSES test-retest correlations ( $(50.84)$ were similar to those of the ASI-R.

TABLE 2. Means and standard deviations of ASI-R and RSES scores for the four groups at baseline, post, and follow-up

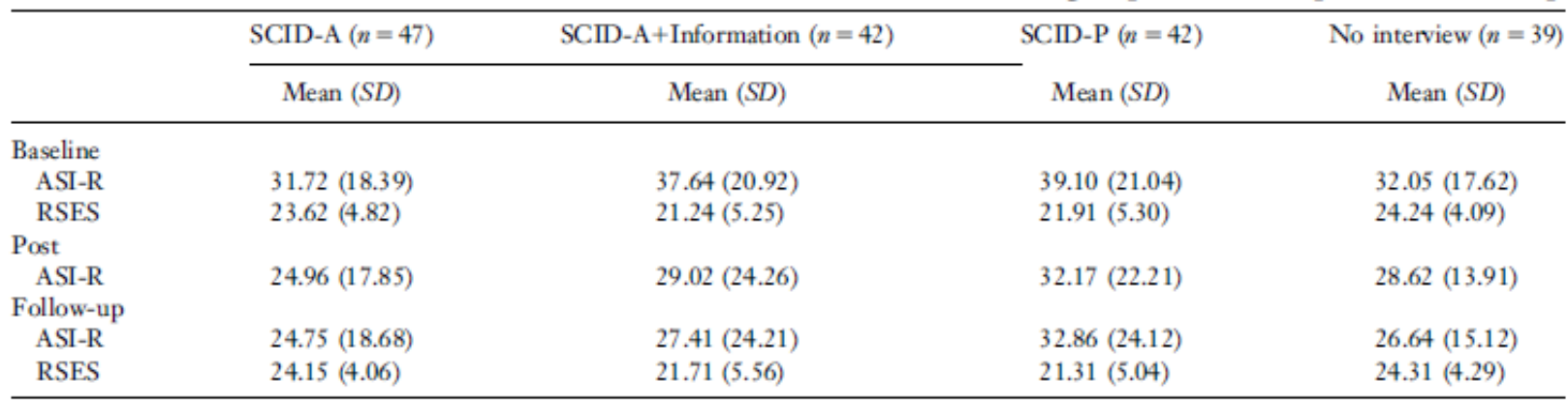

SCID-A, Structured Clinical Interview for DSM-IV Diagnoses-Anxiety Disorders Module; SCID-A+Information, Structured Clinical Interview for DSM-IV Diagnoses-Anxiety Disorders Module plus education regarding physiological responses to anxiety; SCID-P, Structured Clinical Interview for DSM-IV Diagnoses-Psychotic Disorders Module; ASI-R, Anxiety Sensitivity Index-Revised; RSES, Rosenberg Self Esteem Scale.

\section{CHANGES IN OUTCOME MEASURES}

The primary purpose of this study was to examine changes in anxiety sensitivity scores under various conditions. Consequently, the effect of various assessment conditions on anxiety sensitivity, as measured by the ASI-R, was of principal interest. In addition, a measure of self-esteem (the RSES) was included in this study to determine whether any changes in scores found for the ASI-R would be specific to the construct of anxiety sensitivity, or whether general test reactivity could explain the findings.

\section{ANXIETY SENSITIVITY INDEX-REVISED}

Baseline, post, and follow-up means and standard deviations for the ASI-R are presented in Table 2. A 4(group)_3(assessment session) repeated measures ANOVA was computed to determine whether ASI-R scores varied over time as a function of group. Results indicated a significant main effect for the assessment session, $F(2$, 332)553.04, Po.001, Zp 250.24, with higher ASI-R scores at baseline (M535.08, SD519.66) than at post (M528.58, SD519.99) and follow-up (M527.84, SD520.95). Post hoc analyses indicated that the decrease from baseline to post was significant, as was the decrease from baseline to followup. 
However, the decrease from post to follow-up did not reach significance. No main or interaction effect for group was noted (P4.10). Thus, all four groups demonstrated comparable decreases in anxiety sensitivity scores.

TABLE 3. Means and standard deviations of ASI-R scores for individuals with high versus low anxiety sensitivity at baseline, post, and follow-up

\begin{tabular}{lcc}
\hline & $\begin{array}{c}\text { Low anxiety sensitivity } \\
(n=26)\end{array}$ & $\begin{array}{c}\text { High anxiety sensitivity } \\
(n=27)\end{array}$ \\
\cline { 2 - 3 } & Mean (SD) & Mean (SD) \\
\hline $\begin{array}{c}\text { Baseline } \\
\text { ASI-R }\end{array}$ & $10.04(4.62)$ & $68.04(12.51)$ \\
$\begin{array}{l}\text { Post } \\
\text { ASI-R }\end{array}$ & $6.81(5.11)$ & $59.41(22.62)$ \\
$\begin{array}{l}\text { Follow- } \\
\text { up }\end{array}$ & & \\
ASI-R & $6.89(5.40)$ & $57.48(24.72)$ \\
\hline
\end{tabular}

ASI-R, Anxiety Sensitivity Index-Revised.

Although no significant differences were noted between the groups on ASI-R scores at baseline, mean differences between the groups at baseline ranged from 0.33 to 7.38 points. Thus, to ensure that the lack of statistical differences noted between groups on the repeated measures ANOVA was not simply because of differing baseline ASI-R levels, the relative change in ASI-R scores was also compared across the groups. However, results indicated that the groups did not significantly differ with regard to the relative change in ASI-R scores from baseline to post, $F(3,169) 52.31$, P4.05 or from post to follow-up, $F(3,169) 51.34$, P4.05.

\section{HIGH VERSUS LOW ANXIETY SENSITIVITY COMPARISONS}

To examine whether the changes in ASI-R scores could be attributed to regression to the mean, separate repeated measures ANOVAs were conducted on individuals with high (41 SD above the mean) versus low (41 SD below the mean) ASI-R scores. Refer to Table 3 for ASI-R means and standard deviations for the high and low anxiety sensitivity groups at baseline, 
post, and follow-up. Results indicated significant reductions in ASI-R scores for both high (n527), $\mathrm{F}(2,52) 57.90, \mathrm{P} 5.001$, Zp 2 50.23, and low anxiety sensitivity (n526), F(2, 50)511.06, Po.001, Zp 2 50.31, groups. A comparison of the relative change in ASI-R scores indicated that individuals with low anxiety sensitivity reported a $32 \%$ reduction in scores from baseline to post and a 31\% decrease from baseline to follow-up. In contrast, individuals with high anxiety sensitivity only reported a 13 and $16 \%$ decrease in scores from baseline to post and baseline to follow-up, respectively. Thus, reductions in anxiety sensitivity scores appear to occur across the spectrum of anxiety sensitivity, thereby providing evidence against regression to the mean as a potential explanation for the decrease in anxiety sensitivity scores.

\section{ASI-R SUBSCALES}

On the basis of the significant findings with ASI total scores, analyses of the ASI subcomponents were also undertaken in an attempt to precisely identify the components of anxiety sensitivity that are affected by repeated administration of anxiety sensitivity measures. Fear of respiratory symptoms. A 4_3 repeated measures ANOVA was computed on ASI-R fear of respiratory symptoms subscale scores. Results indicated a significant main effect for the assessment session on the fear of respiratory symptoms subscale, $F(2$, 332)568.50, Po.001, Zp 2 50.29, with higher scores at baseline (M515.46, SD59.14) than at post (M511.22, SD58.30) and follow-up (M510.51, SD58.40). Post hoc analyses indicated that the decrease from baseline to post was significant, as was the decrease from baseline to follow-up. However, the change in scores from post to follow-up did not attain significance. A significant group by time interaction effect was also noted, $F(6,332) 52.35$, P5.03, Zp 2 50.04. Follow-up analyses indicated that there was a differential change among the groups in fear of respiratory symptoms from baseline to post, $\mathrm{F}(3$, 169)53.51, P5.02, Zp 2 50.29. Specifically, the no interview group (M51.62, SD55.66) reported significantly less change in fear of respiratory symptoms than the SCID-A plus psychoeducation group (M55.26, SD56.18), and marginally less change than the SCID-A (M54.79, SD55.73) and SCID-P (M55.02, SD55.65). No group differences were 
noted with regard to change from baseline to followup or post to follow-up.

Fear of publicly observable anxiety reactions. A 4_3 mixed model ANOVA of the fear of publicly observable anxiety reactions subscale also revealed a significant main effect for the assessment session, $F(2$, 332)520.29, Po.001, Zp 2 50.11. On average, participant scores significantly decreased from baseline, (M511.96, SD56.40) to post (M510.67, SD57.06) and follow-up (M510.39, SD57.36). However, no main effect for group or interaction effect was noted. Thus, all groups reported comparable decreases in fears of publicly observable anxiety reactions.

Fear of cardiovascular symptoms. A 4_3 mixed model ANOVA of the fear of cardiovascular symptoms subscale indicated a significant main effect for the assessment session, $F(2,332) 54.43$, P5.01, Zp 250.03 . Thus, participants reported a relatively small, though statistically significant, decline in scores from baseline (M55.28, SD55.76) to post (M54.57, SD55.34) and follow-up (M54.72, SD55.55). However, no main effect of group or interaction effect was indicated, suggesting that fears of cardiovascular symptoms decreased regardless of condition.

Fear of cognitive dyscontrol. A 4_3 mixed model ANOVA applied to ASI-R fear of cognitive dyscontrol subscale scores did not indicate any main or interaction effects. Thus, scores on the cognitive dyscontrol domain seem to be relatively stable in comparison with the other ASI-R subscales. ROSENBERG SELF-ESTEEM SCALE In an attempt to determine whether the observed declines in ASI-R scores were the result of test reactivity or a general improvement in mood, a 4_2 repeated measures ANOVA was also computed using RSES scores as the dependent measure. No significant main effect for time was noted, $F(1,166) 50.32$, P5.58, Zp 2 50.002, indicating that mean RSES scores did not change between baseline (M522.74, SD55.00) and follow-up (M522.88, SD54.92), and no group by time interaction was found (P4.20). Thus, overall mean self-esteem scores across the conditions remained stable between baseline and follow-up. The relative change in RSES scores was also not significant among the groups, $F(3,169) 51.44$, P4.20. 


\section{DISCUSSION}

Several recent studies have noted significant reductions in anxiety sensitivity scores in both treatment and control conditions, particularly in the presence of a structured diagnostic interview, raising concerns about the use of self-report anxiety sensitivity measures in prospective research. This study sought to further explore this issue by evaluating the stability of anxiety sensitivity scores under multiple conditions. Overall, results were consistent with those of previous reports in suggesting that anxiety sensitivity scores tend to decline over time. Specifically, scores on a measure of anxiety sensitivity (ASI-R) significantly decreased from baseline to post, regardless of condition, and lower scores were maintained at 2-week follow-up. Thus, regardless of condition, participants, on average, reported significantly less fear of anxiety sensations at the second test administration even if they had not received an interview.

Previous work using individuals with high levels of anxiety sensitivity had indicated that decreases in ASI scores may be due to the administration of a structured interview containing information about anxiety.[9] The rationale behind this idea is that structured interviews may provide indirect normalizing information about anxiety symptoms, which may in turn reduce fears of anxiety-related sensations. We tested this notion experimentally by providing either indirect information (a structured anxiety-related clinical interview), clear and unequivocal direct information (interview plus education), or no information. A comparison of the absolute and relative change in general anxiety sensitivity scores among the indirect information and no interview groups revealed no differences, suggesting that brief normalizing information directly resulting from the administration of a structured diagnostic interview for anxiety disorders cannot account for the systematic declines in overall anxiety sensitivity scores. However, the administration of an anxiety-related diagnostic interview in combination with the provision of direct information regarding the physiological experience of anxiety did appear to convey some benefits. For example, although not statistically significant, a comparison of group means revealed that the direct information group reported a slightly greater decrease in overall anxiety sensitivity scores from baseline to post in comparison with the no interview/ no information group. Furthermore, an analysis of scores on the subcomponents of anxiety sensitivity 
revealed that the provision of direct information did result in significantly greater decreases in fears of respiratory symptoms, from baseline to post, in comparison with the no interview condition. Direct information also produced a greater, though marginally significant, decline in self-reported fear of respiratory symptoms from baseline to post in comparison with indirect information as well. However, it should be noted that these differences were not maintained at 2week follow-up, and no differences were noted between the groups on any of the other subscales. Furthermore, the SCID-A plus education group spent the most time in the laboratory of all the groups, thus raising questions regarding whether the benefits reported by the SCID-A plus education group were due to the educational component or were an artifact of time spent in the laboratory. Thus, although the provision of direct information regarding the dangerousness of anxiety symptoms may convey some additional short-term benefits with regard to fear of respiratory symptoms, these benefits do not appear to generalize to the other facets of anxiety sensitivity, and additional research will be necessary to clarify the source of these benefits.

Noting that previous studies did not investigate the relative latency of the observed anxiety sensitivity reductions, this study sought to address this issue by assessing anxiety sensitivity scores at baseline, immediately after the participant's completion of their relative condition, and again at a 2-week follow-up appointment. Results indicated that the significant reductions in ASI-R scores occurred between baseline and post, a period of approximately 5-7 min on average for the SCID-A, SCID-P, and control conditions, and 25-30 min for the SCID-A plus psychoeducation group. In other words, participants who spent $5 \mathrm{~min}$ alone in a room that contained only chairs and a table reported significant reductions in anxiety sensitivity comparable to those of individuals who spent 30 min completing a diagnostic interview and receiving education regarding typical psychophysiological responses that occur during anxiety responses. Thus, the spontaneous improvements in self-report anxiety sensitivity appear to occur relatively quickly and are maintained for at least several weeks. It will be beneficial for future research to examine the relative duration of these changes in anxiety sensitivity scores, as the time frame under which they occur and are maintained could help to inform the design of anxiety sensitivity research, 
particularly those studies involving a longitudinal assessment of the construct.

Several other potential explanations exist for the decreases in anxiety sensitivity that have now been noted across several studies by independent research groups. For example, it is possible that the mere completion of anxiety sensitivity questionnaires has a systematic impact on self-reported fears of anxiety sensations. It is possible that exposure to questions regarding fears of anxiety sensations leads to increased attention, monitoring, and/or cognitive processing of these fears, which, in turn, may cause a mild, but statistically significant, decrease in anxiety sensitivity. This explanation would be consistent with research indicating that self-monitoring of various other forms of behavior, such as smoking [e.g.,][22] and binge eating,[23] can lead to a significant decline in the monitored behavior. Thus, the mere administration of anxiety sensitivity measures may have a mild effect on fears of anxiety sensations. We are currently in the process of experimentally evaluating this possibility and the potential dose-response effect of administering measures of anxiety sensitivity to individuals over various time frames.

Alternatively, it is possible that heightened levels of arousal or anxiety experienced in anticipation of a novel laboratory situation lead to an artificial inflation of self-reported fears of anxiety sensations. However, as the individual habituates to the laboratory environment and requirements of the study, a reduction in overall arousal and anxiety levels may lead to lower levels of reported fears of anxiety. Although previous research has indicated significant associations between anticipatory anxiety and anxiety sensitivity scores [e.g.,],[24] relatively little is known regarding the influence of anticipatory anxiety on anxiety sensitivity scores. Thus, it may be beneficial for future research into the spontaneous decline in anxiety sensitivity scores to assess for anticipatory anxiety via self-report and physiological measures to determine whether changes in anticipatory anxiety are associated with concomitant changes in anxiety sensitivity.

A third potential explanation for these findings is that statistical regression, test reactivity, or some other nonspecific effect of the experimental design led participants to respond to the questionnaires in a more positive manner at post and follow-up. In an attempt to reduce the impact of statistical regression in this study, 
participants were not pre-selected based on extreme scores. Rather, individuals representing the full range of anxiety sensitivity scores were included in this study, and baseline ASI-R scores were found to be comparable to other non-selected samples [e.g.,].[11] Furthermore, a direct comparison of individuals with high [ASI-R scores $41 \mathrm{SD}$ ) versus low (ASI-R scores o1 $\mathrm{SD})$ anxiety sensitivity revealed that both groups reported significant reductions in anxiety sensitivity, with the low anxiety sensitivity individuals demonstrating a greater decline in relative change scores. Thus, it is highly unlikely that the observed decreases in anxiety sensitivity scores are the result of selection biases or statistical regression.

To determine whether the temporal instability in self-report anxiety sensitivity scores was merely reflective of a general reactivity to assessment instruments, a non-anxiety sensitivity measure was also administered to participants. Results indicated that participants' scores on a measure of self-esteem, which is a related but conceptually distinct construct, did not evidence significant changes over time. Thus, the temporal instability in anxiety sensitivity scores does not appear to be part of a broad-based phenomenon; but rather is specific to verbal-report measures of anxiety sensitivity. Replication of the present findings with other selfreport measures of anxiety sensitivity [e.g., anxiety sensitivity profile],[25] along with an analysis of the stability of other anxiety sensitivity-related constructs (e.g., trait anxiety), would help to confirm that the often observed decrease in anxiety sensitivity scores is indeed specific to verbal-report measures of anxiety sensitivity.

Although spontaneous declines in anxiety sensitivity scores have been noted in numerous studies and the results of this research suggest that this effect is a relatively robust phenomenon, we should keep in mind that these investigations have relied on a single mode of assessment (i.e., self-report ASI or ASI-R scores). Thus, whether these declines in verbal-report anxiety sensitivity scores are truly reflective of decreased fear of anxiety symptoms, or whether they are merely indicative of the temporal instability of anxiety sensitivity self-report measures, remains unknown. Additional research using alternative indicators of anxiety sensitivity (e.g., physiological responses to biological challenge procedures) may prove useful in determining the extent to which spontaneous reductions in verbal report anxiety sensitivity scores are associated with 
concurrent changes in the physiological and behavioral manifestations of anxiety sensitivity.

Regardless of the source, the now well-replicated phenomenon of declining anxiety sensitivity scores provides an interesting cautionary tale for clinical researchers. Specifically, a systematic decline in anxiety sensitivity scores has critical implications for the design and interpretation of longitudinal research. For example, this research highlights the importance of demonstrating that treatments designed to impact anxiety sensitivity, at a minimum, produce significantly greater reductions in anxiety sensitivity than control conditions assessed over the same time frame. Although numerous anxiety sensitivity interventions have been investigated, ranging from physical exercise[26] to cognitive-behavioral therapy [e.g.,],[27] many of the efficacy studies supporting these interventions either have not included an appropriate control condition [e.g.,],[27,28] or have not demonstrated the intervention to be significantly more effective in reducing anxiety sensitivity than control [e.g.,].[7] In addition to ensuring the use of adequate control conditions, researchers should consider including multiple baseline measurements of anxiety sensitivity in their prospective research designs. The results of this study suggest that the decline in anxiety sensitivity scores among ostensible control conditions occurs relatively early (i.e., within approximately 5-30 min of completing the anxiety sensitivity measure the first time), and then remains relatively stable. Thus, multiple baseline measurements may provide researchers with a way to control for the "natural" decline in anxiety sensitivity scores that has now been observed across multiple studies and various research designs.

Perhaps most importantly, these findings have important implications for research beyond the anxiety sensitivity field. Psychometric studies of rating scales usually report temporal stability using simple bivariate correlations conducted on two administrations of an instrument. Results of this study, in which we found good test-retest correlations but also significant changes in means, suggest that a fuller picture of an instrument's psychometric characteristics requires a comparison of means across administrations. Although not currently a standard practice, behavioral scientists should be encouraged to include this basic information when reporting the temporal properties of an instrument. 


\section{REFERENCES}

1. Reiss S, McNally RJ. The expectancy model of fear. In: Reiss S, Bootzin RR, editors. Theoretical issues in behavior therapy.

London: Academic Press; 1985.

2. Maller RG, Reiss S. Anxiety sensitivity in 1984 and panic attacks in 1987. J Anxiety Disord 1992;6:241-247.

3. Schmidt NB, Lerew DR, Jackson RJ. The role of anxiety sensitivity in the pathogenesis of panic: prospective evaluation of spontaneous panic attacks during acute stress. J Abnorm Psychol 1997;106:355-364.

4. Peterson RA, Reiss S. Anxiety Sensitivity Index revised manual. Worthington, $\mathrm{OH}$ : International Diagnostic Systems Publishing Corporation; 1992.

5. Weems CF, Hayward C, Killen J, Taylor CB. A longitudinal investigation of anxiety sensitivity in adolescence. J Abnorm Psychol 2002;111:471-477.

6. Zinbarg RE, Schmidt NB. Evaluating the invariance of the structure of anxiety sensitivity over five weeks of basic cadet training in a large sample of Air Force cadets. Pers Individ Differences 2002;33:815-832.

7. Gardenswartz CA, Craske MG. Prevention of panic disorder. Behav Ther 2001;32:725-737.

8. Schmidt NB, Eggleston AM, Woolaway-Bickel K, Fitzpatrick KK, Vasey MW, Richey JA. Anxiety Sensitivity Amelioration Training (ASAT): a longitudinal primary prevention program targeting cognitive vulnerability. J Anxiety Disord 2007;21: 302-319.

9. Maltby N, Mayers MF, Allen GJ, Tolin DF. Anxiety sensitivity: stability in prospective research. J Anxiety Disord 2005;19: 708-716.

10. Taylor S, Cox BJ. An expanded Anxiety Sensitivity Index: evidence for a hierarchic structure in a clinical sample. J Anxiety Disord 1998a;12:463-483.

11. Deacon BJ, Abramowitz JS, Woods CM, Tolin DF. The Anxiety Sensitivity Index- Revised: psychometric properties and factor structure in two nonclinical samples. Behav Res Ther 2003;41:1427-1449. 
12. Rosenberg M. Society and the adolescent self-image, revised edition. Middletown, CT: Wesleyan University Press; 1989.

13. Marsic A, Broman-Fulks JJ, Harris JA, Martin HM. Anxiety sensitivity, self-esteem, and mood. Poster presented at the 40th annual convention of the Association for Behavior and Cognitive Therapies, Chicago, IL; 2006.

14. Fleming JS, Courtney BE. The dimensionality of self-esteem: II. Hierarchical facet model for revised measurement scales. J Pers Soc Psychol 1984;46:404-421.

15. Blascovich J, Tomaka J. Measures of self-esteem. In: Robinson JP, Shaver PR,Wrightsman LS, editors. Measures of personality and social psychological attitudes (3rd ed.). Ann Arbor: Institute for Social Research; 1993:115-160.

16. Rosenberg M. Conceiving the self. Malabar, FL: Krieger; 1986.

17. First MB, Spitzer RL, Gibbon M, Williams JBW. Structured clinical interview for DSM-IV-TR Axis I disorders, research version, Non-patient Edition. (SCID-I/NP). New York: Biometrics Research, New York State Psychiatric Institute; 2002.

18. Zanarini MC, Skodol AE, Bender D, Dolan R, Sanislow C, Schaefer E, Morey LC, Grilo CM, Shea MT, McGlashan TH, Gunderson JG. The Collaborative Longitudinal Personality Disorders Study: reliability of axis I and II diagnoses. J Personal Disord 2000;14:291-299.

19. Skyre I, Onstad S, Torgersen S, Kringlen E. High interrater reliability for the Structured Clinical Interview for DSM-III-R Axis I (SCID-I). Acta Psychiatr Scand 1991;84:167-173.

20. Barlow DH, Craske MG. Mastery of your anxiety and panic (MAP-3): client workbook for anxiety and panic. Oxford: Oxford University Press; 1999.

21. Deacon B, Abramowitz J. Anxiety and its dimensions across the anxiety disorders. J Anxiety Disord 2006;20:837-857.

22. Kantorowitz DA, Walters J, Pezdek K. Positive versus negative self-monitoring in the self-control of smoking. J Consult Clin Psychol 1978;46:1148-1150.

23. Latner JD, Wilson GT. Self-monitoring and the assessment of binge eating. Behav Ther 2002;33:465-477. 
24. Vujanovic AA, Zvolensky MJ, Gibson LE, Lynch TR, Leen-

Feldner EW, Feldner MT, Bernstein A. Affect intensity:

association with anxious and fearful responding to bodily

sensations. J Anxiety Disord 2006;20:192-206.

25. Taylor S, Cox BJ. Anxiety sensitivity: multiple dimensions and hierarchic structure. Behav Res Ther 1998;36:37-57.

26. Broman-Fulks JJ, Berman ME, Rabian B,Webster MJ. Effects of aerobic exercise on anxiety sensitivity. Behav Res Ther 2004;42: 125-136.

27. Penava SJ, Otto MW, Maki KM, Pollack MH. Rate of improvement during cognitive-behavioral group treatment for panic disorder. Behav Res Ther 1998;36:665-673.

28. Otto MW, Pollack MH, Fava M, Uccello R, Rosenbaum JF. Elevated Anxiety Sensitivity index scores in patients with major depression: correlates and changes with antidepressant medication. J Anxiety Disord 1995;9:117-123. 